\title{
Increasing Incidence of Extended-Spectrum Beta-Lactamase-Producing Enterobacteriaceae (ESBL) and the Relation to Consumption of Broad-Spectrum Antimicrobial Agents 2003-2011 in a Large Area of Copenhagen, Denmark
}

\author{
Lene Nielsen ${ }^{*}$, Anne Kjerulf ${ }^{1,2}$, Magnus Arpi ${ }^{1}$ \\ ${ }^{1}$ Department of Clinical Microbiology, Copenhagen University Hospital, Herlev, Denmark \\ ${ }^{2}$ Department of Microbiology and Infection Control at Statens Serum Institut, Copenhagen, Denmark \\ Email: ${ }^{*}$ lene.nielsen.02@heh.regionh.dk
}

Received 17 February 2015; accepted 3 March 2015; published 5 March 2015

Copyright (C) 2015 by authors and Scientific Research Publishing Inc.

This work is licensed under the Creative Commons Attribution International License (CC BY).

http://creativecommons.org/licenses/by/4.0/

(c) (i)

Open Access

\begin{abstract}
Purpose: To investigate 1) the development in the incidence of ESBL-producing bacteria in hospitals and primary health care, 2) the contribution of primary health care to the incidence of ESBLproducing bacteria, and 3) the development in resistance patterns for all Escherichia coli and Klebsiella pneumoniae isolates in relation to antimicrobial consumption in hospitals and primary health care. Methods: ESBL-data were retrospectively collected from bacterial isolates from all specimens received at the Department of Clinical Microbiology from 2003 to 2011 together with the corresponding patient data. ESBL-production was detected in isolates from 1067 of 59,373 patients (1.8\%) with an E. coli infection and in 263 of 8660 patients (3.0\%) with a $K$. pneumoniae infection. Results: From 2003 to 2009, an increase in patients with an ESBL-producing isolate occurred in both hospitals and primary health care at the same time as an increased consumption of broad-spectrum antimicrobial agents was seen. Interventions to reduce prescription of cephalosporins and ciprofloxacin at the hospitals from 2010 resulted in a remarkable decrease in patients with ESBL-producing $K$. pneumoniae whereas a continuing increase was seen in patients with ESBL-producing $E$. coli both at hospitals and in primary health care. The proportion of patients with community-acquired ESBL-producing $E$. coli was stable with an increase of only $1.4 \%$ from 2007 to 2011. Conclusions: Reduction in prescription of broad-spectrum antimicrobial agents
\end{abstract}

${ }^{*}$ Corresponding author.

How to cite this paper: Nielsen, L., Kjerulf, A. and Arpi, M. (2015) Increasing Incidence of Extended-Spectrum Beta-Lactamase-Producing Enterobacteriaceae (ESBL) and the Relation to Consumption of Broad-Spectrum Antimicrobial Agents 2003-2011 in a Large Area of Copenhagen, Denmark. Open Journal of Medical Microbiology, 5, 28-42.

http://dx.doi.org/10.4236/ojmm.2015.51005 
at the hospital level had an important impact on the incidence of ESBL-producing $K$. pneumoniae, but not on ESBL-producing E. coli.

\section{Keywords}

Incidence of ESBL-Producing E. coli and K. pneumoniae, Hospital Care, Primary Health Care, Antimicrobial Consumption, Broad-Spectrum Antimicrobial Agents

\section{Introduction}

The increasing frequency of extended spectrum beta-lactamase (ESBL)-producing Enterobacteriaceae in recent years is of great concern worldwide [1]-[3]. ESBL-producing Enterobacteriaceae are resistant to all penicillins, cephalosporins and monobactams. Furthermore, they are inhibited by clavulanic acid, placing them in the functional group 2be of the Bush-Jacoby-Medieros classification system [4] [5]. The ESBL-genes are located on plasmids which also often carry genes coding for resistance to aminoglycosides and fluoroquinolones; these plasmids can easily be transferred between Enterobacteriaceae, especially E. coli and K. pneumoniae [1]-[5]. The CTX-M enzymes are the most widespread ESBL-enzymes, and several outbreaks have been reported in recent years, most caused by clonal spread of CTX-M-15-producing K. pneumoniae strains in the hospitals [6] and E. coli strains in the community [7]. Small hospital outbreaks have primarily been reported from the Scandinavian countries with mainly CTX-M-15 K. pneumoniae, but there has been one large hospital outbreak in Uppsala, Sweden from 2005 to 2007 with more than 300 cases [8].

In Denmark, there is no mandatory reporting and no national surveillance of ESBL-producing Enterobacteriaceae, but nationwide prevalence studies in 2007, 2009, and 2011 have revealed high prevalences of ESBLproducing isolates, being much higher than the incidences seen in Norway and Sweden [9]-[12]. The Scandinavian countries have generally had a lower consumption of broad-spectrum antimicrobial agents compared to Europe as a whole [13]. The consumption of these antimicrobial agents has, however, increased with $70 \%$ from 2002 to 2011 in Denmark [10]. A decrease in consumption of broad-spectrum antimicrobial agents has been documented from 2009 to 2011 in hospitals, but an increase is still on-going in primary health care with the highest observed consumption in 2011 since reporting from DANMAP (The Danish Integrated Antimicrobial Resistance Monitoring and Research Programme) began in 1995 [10].

This is the first Danish study looking at both the incidence of ESBL-producing E. coli and K. pneumoniae and the consumption of antimicrobial agents in primary health care and hospital care during a period of nine years from 2003 to 2011.

The aims of this study were to investigate 1) the development in the incidence of ESBL-producing bacteria from 2003 to 2011 in hospitals and primary health care in the Copenhagen area referring to the Department of Clinical Microbiology at Herlev Hospital, 2) the contribution from primary health care to the incidence of ESBL-producing bacteria, 3) the development in antimicrobial resistance patterns of E. coli and K. pneumoniae isolates in relation to the antimicrobial consumption in the hospitals in general, in selected wards, and in primary health care.

\section{Patients and Methods}

\subsection{Susceptibility Testing and ESBL-Testing Methods Employed}

Antibiotic susceptibility testing was until October 2009 done by the disk diffusion method using Oxoid $^{\mathrm{TM}}$ disks (Basingstoke, UK) with semi-confluent growth on Iso-sensitest agar without blood (Substrate Department, Herlev University Hospital) and with breakpoints according to SRGA (The Swedish Reference Group for Antibiotics) [14]. After this date susceptibility testing was done using mueller hinton agar (Substrate Department, Herlev University Hospital) with confluent growth and breakpoints according to EUCAST (The European Committee on Antimicrobial Susceptibility Testing) [15]. The following agents were tested: ampicillin, mecillinam, cefuroxime, gentamicin, tobramycin, sulfamethizole, trimethoprim, nitrofurantoin, ciprofloxacin, meropenem, and cefpodoxime. Piperacillin-tazobactam was not routinely used before 2011. 
Various ESBL-testing methods were used during the study period, as shown in Table 1. Until October 2009 only clavulanic acid was used as inhibitor of beta-lactamases, thereby missing detection of the AmpC-enzyme and isolates containing both ESBL and AmpC. From October 2009 a new method using the Mast group ABCD [16] was introduced in the laboratory. This consists of different combinations of cefpodoxime, clavulanic acid and/or cloxacillin enabling detection of isolates with ESBL- and/or AmpC-enzymes. Isolates only containing AmpC-enzymes were not included in 2010 and 2011 in order to compare the development in incidences with the previous years.

\subsection{Collection of ESBL Data}

From 2003 to 2010, the Department of Clinical Microbiology at Herlev Hospital served the former Copenhagen county area with three university hospitals and one district hospital with a population of approximately 620,000 in the catchment area. From January 2011 this was reduced to two university hospitals and a population of 423,000 in the new catchment area.

Data were collected from all specimens received at the Department of Clinical Microbiology in the study period. All phenotypically ESBL-producing isolates of E. coli and K. pneumoniae with antimicrobial susceptibilities were extracted from the laboratory data system. The following data were collected from the database: age and sex of patients, hospital versus community acquired infection, hospital ward, type of specimen, and relation between detection of the first ESBL isolate to current or prior hospitalization. The resistance patterns of the ESBL isolates were compared to the resistance patterns of the non-ESBL E. coli and K. pneumoniae isolates from the same time period.

For the ESBL-producing isolates only one isolate per patient per year was included. For the non-ESBL isolates all isolates were included. The extraction of one isolate per patient per year was done manually, and due to the high number of patients with non-ESBL isolates this was not possible for these isolates.

\subsection{Distinction between Community-Acquired and Health Care Associated Infections}

As fecal carriage of ESBL presumably precedes most cases of ESBL-infections, the usual definitions of infections as hospital or community acquired may not be valid. In order to obtain a more realistic view of the number of patients with true community acquired ESBL-producing isolates, we categorized patients according to the time elapsed between their latest hospital discharge and the first finding of an ESBL-producing isolate from them. The following seven categories were defined: acquisition during hospitalization, acquisition within 3 months, 3 - 6 months, 6 - 12 months, 1 - 2 years, 2 - 5 years, and later than 5 years after discharge or not at all. Hospital admissions lasting only one day were not included. These alternative definitions of hospital acquired ESBL-infections (hospitalization within two years before the first finding of an ESBL isolate) and community acquired ESBL-infections (discharge from hospital two years after the first finding of an ESBL isolate) are discussed below.

\subsection{Antimicrobial Consumption in Hospital Care and Primary Health Care}

Data were provided from the Hospital Pharmacy at Herlev Hospital and from the Danish Health and Medicines Authority for Primary Health Care in the Copenhagen area. Data were provided as defined daily dose (DDD)/ year, as the total number of bed days was not available for all the hospitals, but only the medical wards.

\section{Results}

\subsection{Demographic Data for ESBL Isolates and Patients with an ESBL Isolate}

The total number of patients with an E. coli isolate for all nine years was 59,373, with a mean of 6597 patients per year. Overall 1067 patients had an infection with an ESBL-producing E. coli during the study period: 691 women and 376 men with a mean age of 63 years and a range of 2 months to 100 years.

The total number of patients with a K. pneumoniae isolate was 8660, with a mean of 962 patients per year. Overall 263 patients had an infection with an ESBL-producing K. pneumoniae during the study period: 135 women and 128 men with a mean age of 71 years and a range of 1 to 98 years.

Table 2 shows the distribution of ESBL isolates and patients with an ESBL isolate, respectively, in various 


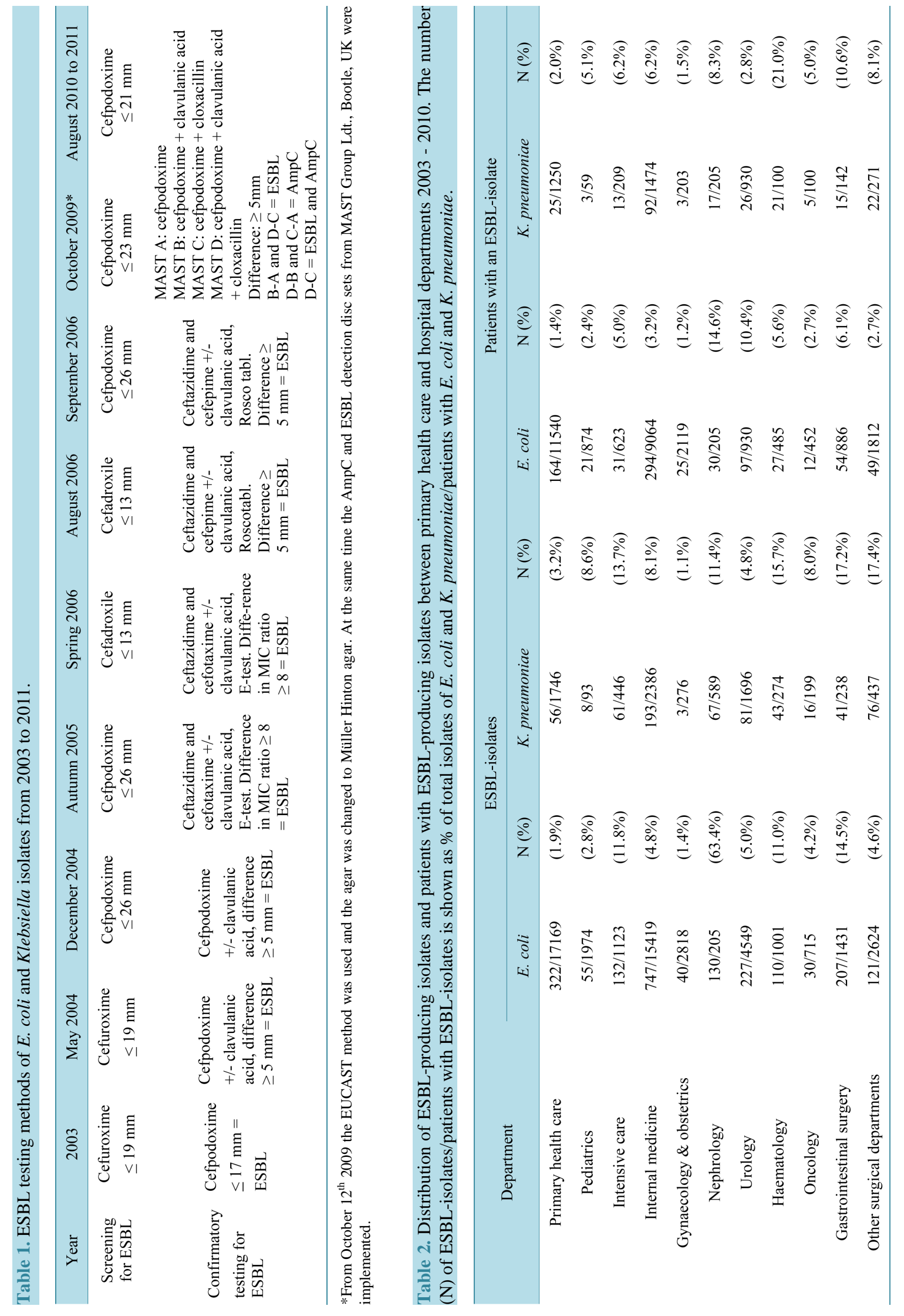


departments and in primary health care from 2003 to 2010. As seen, there was a high incidence of ESBL-producing strains/patients with an ESBL isolate in the departments of haematology, nephrology, urology, gastrointestinal surgery, intensive care and internal medicine.

\subsection{ESBL-Testing Methods}

The application of the new four-disc method that detects both AmpC and ESBL in 2010 resulted in the finding of two out of $167 \mathrm{~K}$. pneumoniae isolates with both AmpC and ESBL (1.2\%) in 2010 and two of 123 (1.6\%) in 2011. For ESBL-positive $E$. coli isolates there were 17 out of 505 isolates having both resistance mechanisms in 2010 (3.4\%) and 19 out of 568 isolates in 2011 (3.3\%).

\subsection{Resistance Patterns in ESBL-Producing and Non ESBL-Producing Isolates}

Table 3 shows the development in resistance for hospitals and primary care combined in ESBL-producing and non-producing isolates towards the commonly used antimicrobial agents in Denmark. Only one ESBL isolate per patient per year was included.

There are major differences between ESBL-producing and non-producing E. coli isolates regarding percentage of resistant isolates, as seen in Table 3(a). All ESBL isolates are inherently resistant to ampicillin, but there was an increase in resistance towards this agent in non-ESBL isolates from 41\% in 2003 to $47 \%$ in 2011. For ciprofloxacin there was an increase from $7 \%$ to $17 \%$ in non-ESBL isolates and from $33 \%$ to $68 \%$ (76\% in 2009) in ESBL isolates during the study period. High and almost stable resistances throughout the study period was seen to sulfamethizole and trimethoprim; in non-ESBL isolates resistance was 36\% - 40\% for sulfamethizole, in ESBL isolates $66 \%-84 \%$. Trimethoprim resistance was $24 \%-28 \%$ in non-ESBL isolates and $63 \%-77 \%$ in ESBL isolates. Resistance to gentamicin was low and stable during the nine years in non-ESBLs (1.5\% - 5\%), whereas it was higher and stable around 33\% - 46\% in ESBLs from 2006 to 2011. Resistance to tobramycin was on the same low level as gentamicin in non-ESBLs, but lower than gentamicin in ESBLs (24\% - 39\%) from 2006 to 2011.

In non-ESBLs, resistance to mecillinam was low (4\% - 8\%), whereas in ESBLs there are only data for 2010 (10\%) and 2011 (9\%). Before that time mecillinam was routinely reported as resistant in ESBL-producing isolates. As mentioned above, isolates were not routinely examined for resistance towards piperacillin-tazobactam before 2011; the percentage of $E$. coli isolates resistant to piperacillin-tazobactam was 5\% for ESBL-producing isolates and 2.3\% for non-ESBL isolates in 2011 (data not shown).

As shown in Table 3(b), there are also major differences in ESBL and non-ESBL $K$. pneumoniae isolates regarding percentage of resistant isolates, but the resistance pattern is similar to $E$. coli regarding most of the antimicrobial agents, e.g. ciprofloxacin, sulfamethizole, and trimethoprim. Due to a small number of ESBL isolates $(<10)$ in 2003-2005, only results from 2006 to 2011 are reported. Resistance to gentamicin and tobramycin has been low and stable during the nine years in non-ESBLs ( $0 \%-6 \%)$, whereas it has been high and increasing in ESBLs from 50\% in 2006 to 65\% in 2011 for gentamicin and from 30\% in 2006 to 53\% in 2011 for tobramycin. Compared to E. coli there is a high resistance towards nitrofurantoin both in ESBL (30\% in 2011) and nonESBL isolates (22\% in 2011). In 2011 the percentage of K. pneumoniae isolates resistant to piperacillin-tazobactam was $8.3 \%$ for ESBL-producing isolates and $0.3 \%$ for non-ESBL isolates (data not shown).

\subsection{Trends in Occurrence of Patients with ESBL Isolates from 2003 to 2011}

The proportion of patients with an ESBL-producing isolate in relation to the total number of patients with an infection due to E. coli (Figure 1(a)) and K. pneumoniae (Figure 1(a)) has been steadily increasing from 2003 to 2011. However, from 2010 to 2011 there has been a small decrease in the incidence of patients with an ESBLproducing K. pneumoniae isolate, especially in hospital-acquired cases. The same tendency was not observed for patients with an ESBL-producing $E$. coli isolate where a continuing increase was seen both in hospitals and in primary health care. There was a remarkable increase in the proportion of ESBL-producing E. coli in patients with bacteraemia compared to the total number of patients with an ESBL-producing E. coli. This phenomenon was not observed for ESBL-producing K. pneumoniae. The total number of new patients with an ESBL-producing $E$. coli increased from 22 in 2003 to 261 in 2011. The total number of new patients with an ESBL-producing K. pneumoniae increased from 6 in 2004, when it was lowest, to 52 in 2010 with a decrease to 43 in 2011. 


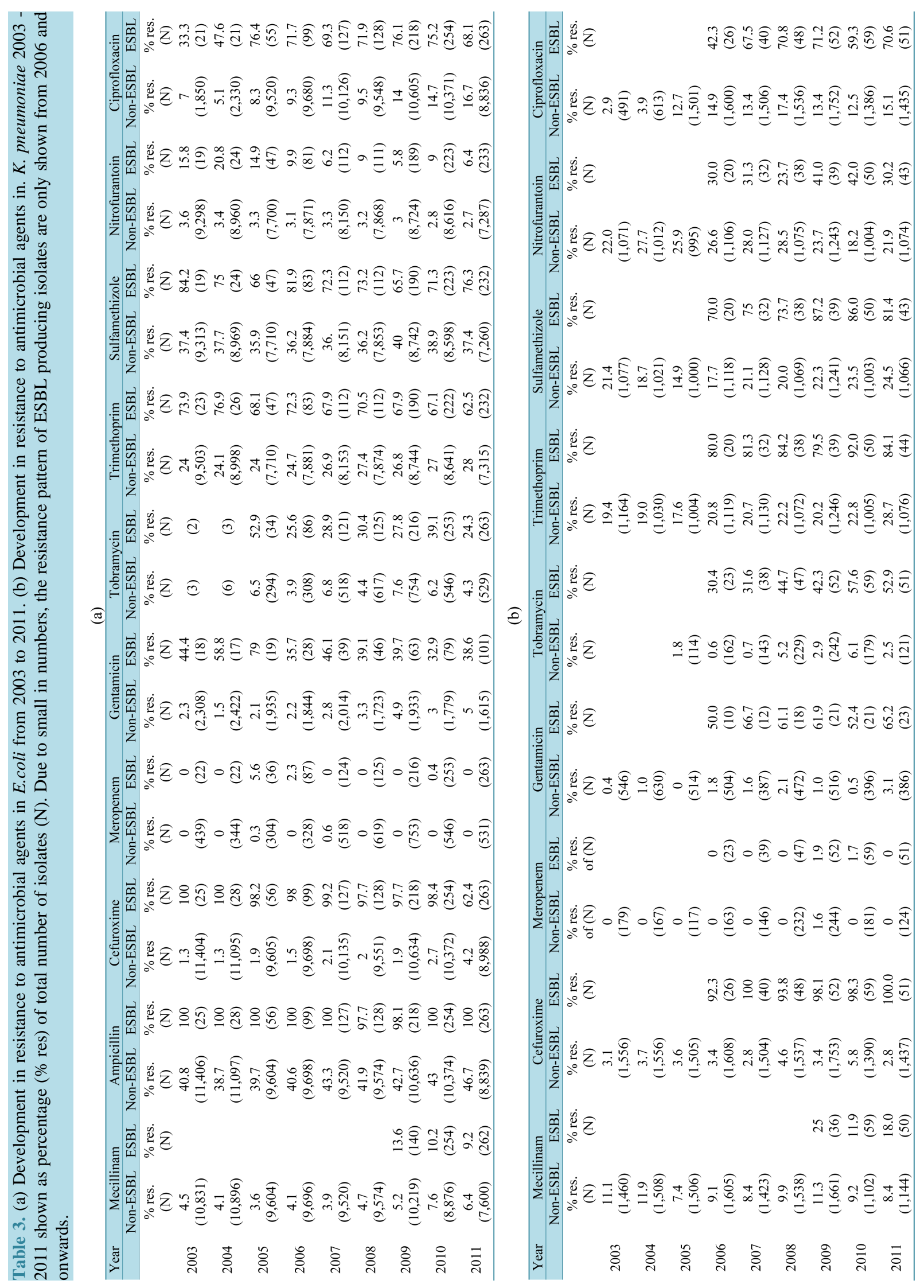




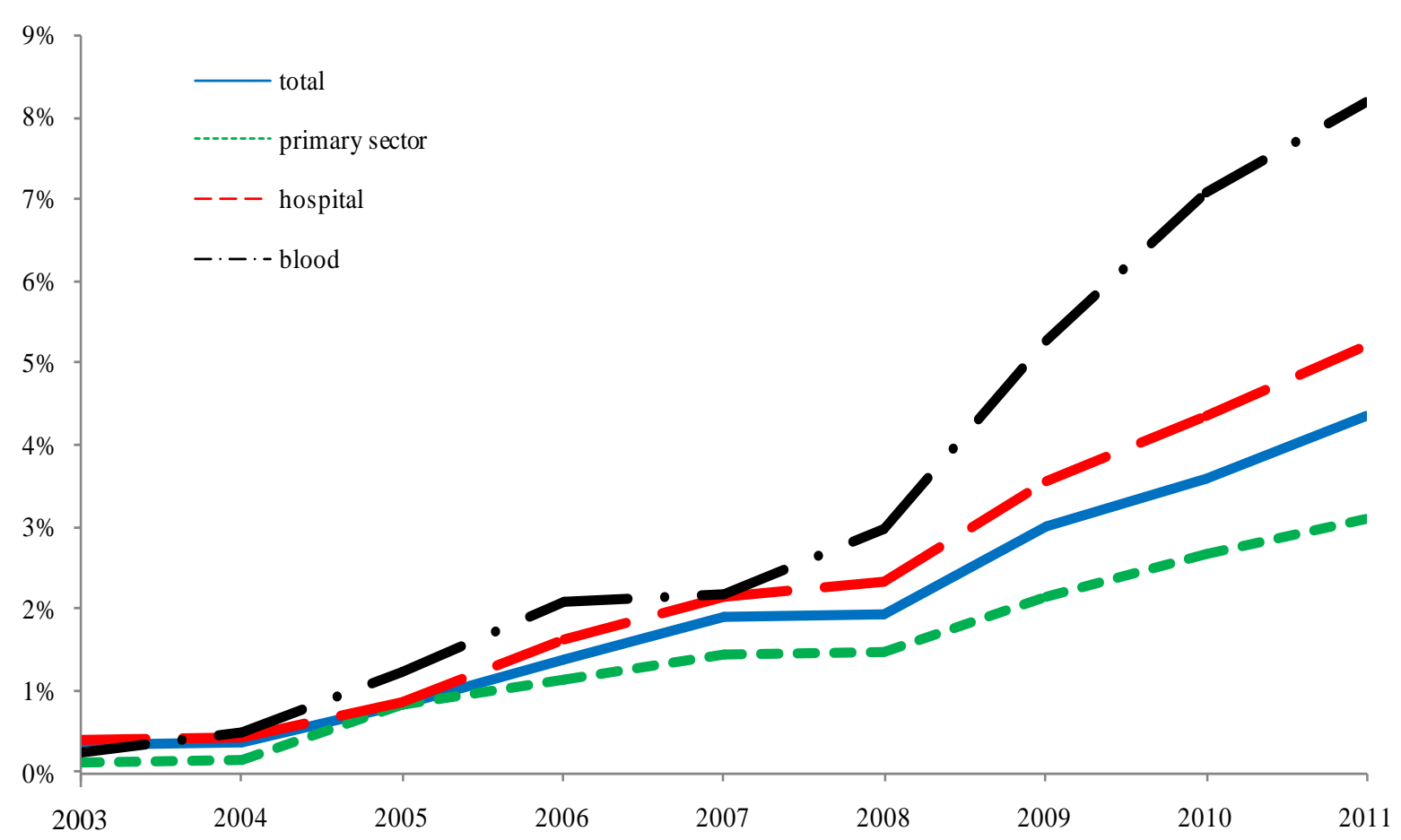

(a)

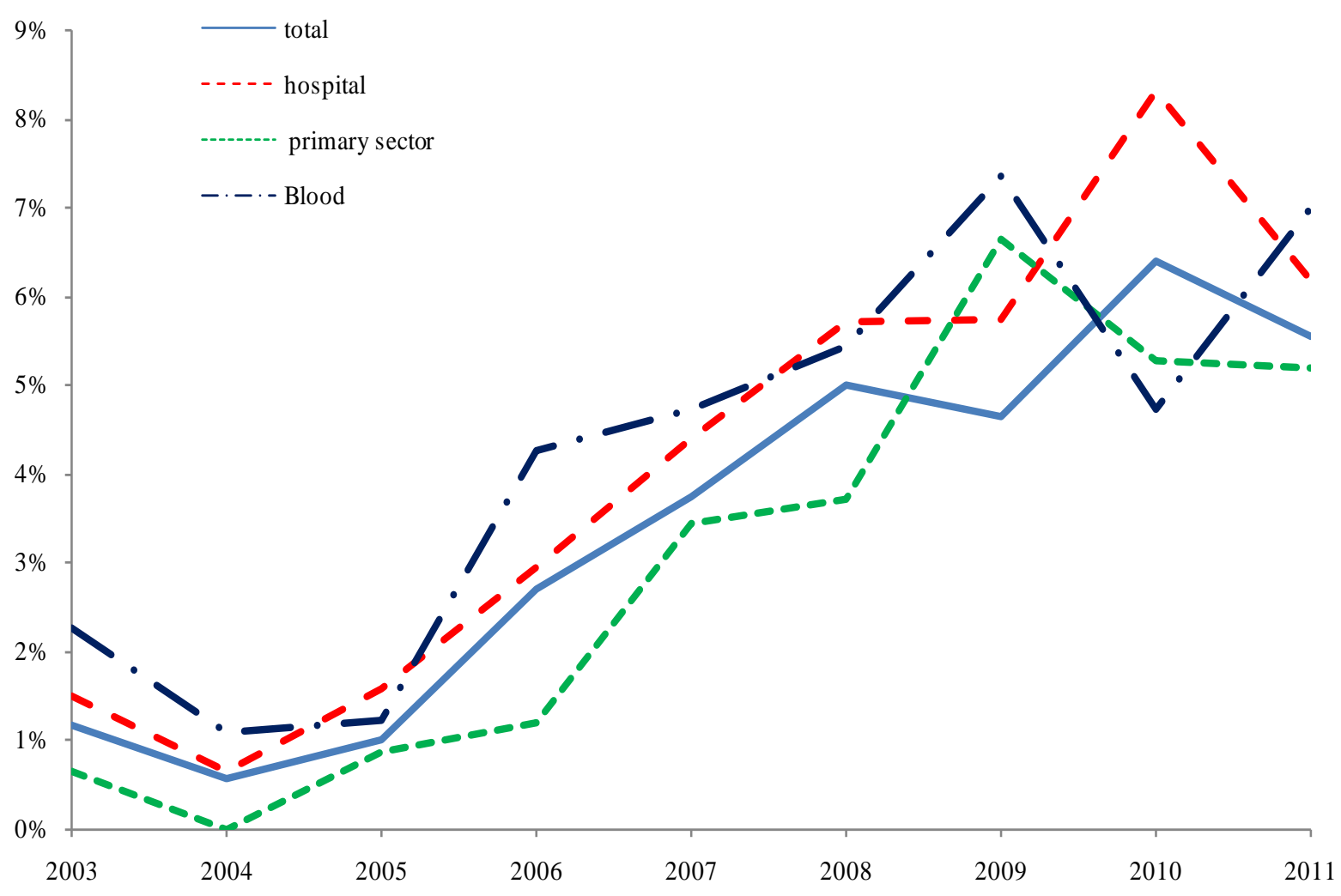

(b)

Figure 1. The proportion of patients per year with an ESBL-producing isolate in relation to the total number of patients with an infection with E. coli (a) and K. pneumoniae (b). Blue line: all patients with an ESBL isolate; green line: all patients with isolates taken in primary health care; red line: all patients with isolates taken at the hospital; black line: isolates from blood culture. 
Figure 2(a) shows the annual distribution of patients according to hospitalization status when their first ESBL-producing isolate was detected. The proportion of patients with their first ESBL-producing E. coli detected during hospitalization declined from $42 \%$ in 2005 to $21 \%$ in 2011. If patients who were discharged from hospital within three months after detection of their first ESBL-producing isolate were included in the group acquiring their first ESBL isolate during hospitalization, the decline from 2005 to 2011 would only be from 52\% to $47 \%$. This indicates that the above mentioned difference could be due to patients being discharged earlier at the end than at the beginning of the study period and not due to a regular decrease in hospital acquired incidence. The proportion of patients never hospitalized grew from $19.8 \%$ in 2005 to $24.1 \%$ in 2011. If patients belonging to the group "not hospitalized within 2 years before presenting with an ESBL isolate" were added to patients never hospitalized, the incidence was stable from 31.1\% in 2005 to $30.6 \%$ in 2011). In 2007 and onwards the total number of new ESBL-patients per year had increased to above 100. From 2007 a small linear increase was observed in the proportion of new ESBL-patients who were not hospitalized within the last two years before detection of their first ESBL-producing isolate. The proportion increased from $29.3 \%$ in 2007 to $30.7 \%$ in 2011 (regression coefficient of 1.01; data not shown) and may represent the increasing contribution of new ESBL-patients from primary health care. The median hospitalization time for those patients who presented with their first ESBL-producing E. coli during hospitalization was 17 days (range 3 - 185 days; data not shown).

Figure 2(b) shows the same data for ESBL-producing K. pneumoniae as for E. coli. The proportion of new patients presenting with an ESBL isolate while hospitalized was 81.8\% in 2003, declining to 50\% in 2004 and to $34.9 \%$ in 2011. If new patients presenting with an ESBL-producing $K$. pneumoniae within three months after discharge are included in the hospitalized group, the incidences are much higher, from $100 \%$ in $2003,83.3 \%$ in 2004 to $67.5 \%$ in 2011. For patients who had not been hospitalized the actual numbers are small and fluctuating from $17.4 \%$ in 2006 to $6.5 \%$ in 2009 . If patients not hospitalized within two years were added to patients never hospitalized the incidences were lower, but still differing from $17.4 \%$ in 2006 to $8.7 \%$ in 2009. For K. pneumoniae there was no linear association with any of the above-mentioned groups. The median hospitalization time for those patients who presented with their first ESBL-producing $K$. pneumoniae isolate during hospitalization was 23 days (range 3 - 139 days; data not shown).

\subsection{Antimicrobial Consumption and ESBL-Producing Isolates}

The overall consumption of antimicrobial agents and incidence of ESBL-producing E. coli and K. pneumoniae from 2003 to 2011 in hospital care and in primary health care can be seen in Figure 3 and Figure 4, respectively. As illustrated in Figure 3, there has been an increase in the total consumption of cephalosporins and fluoroquinolones from 2003 to 2009 and at the same time an increase in incidence of ESBL-producing isolates, especially K. pneumoniae. In 2010 a decrease in consumption of these antimicrobial agents was followed by a decrease in ESBL-producing $K$. pneumoniae but not in ESBL-producing $E$. coli. There has been an increased consumption of piperacillin-tazobactam, mecillinam and meropenem from 2003 to 2010. During the same time span there has been a decreased consumption of gentamicin, sulfamethizole, and trimethoprim.

Figure 4 shows that there has been an increased consumption of mecillinam, amoxicillin with clavulanic acid, nitrofurantoin, and trimethoprim in the primary sector from 2003 to 2010. At the same time there has been a decrease in consumption of pivampicillin and sulfamethizole. An increased use of ciprofloxacin was seen until 2007 but has since declined. An increase in incidence of ESBL-producing isolates, especially in K. pneumoniae, has occurred until 2009. From 2009 there has been a decrease in ESBL-producing K. pneumoniae isolates, but not in ESBL-producing E. coli isolates.

The Department of Internal Medicine at Herlev Hospital with a high incidence of ESBL-producing strains initially had a high consumption of broad-spectrum antimicrobial agents/1000 bed days; but a gradual reduction in the use of fluoroquinolones and cephalosporins during 2009-2011 was followed by a decrease in ESBL-strains, especially K. pneumoniae, as illustrated in Figure 5. The use of fluoroquinolones and cephalosporins declined and at the same time the use of piperacillin-tazobactam and to a lesser extent meropenem increased. The same tendency was also seen at other departments.

\section{Discussion}

In a few years, Denmark went from being a low to being a high prevalence country with regard to ESBL-producing bacteria. The first sporadic cases were described in 1999, both patients having been hospitalized abroad 


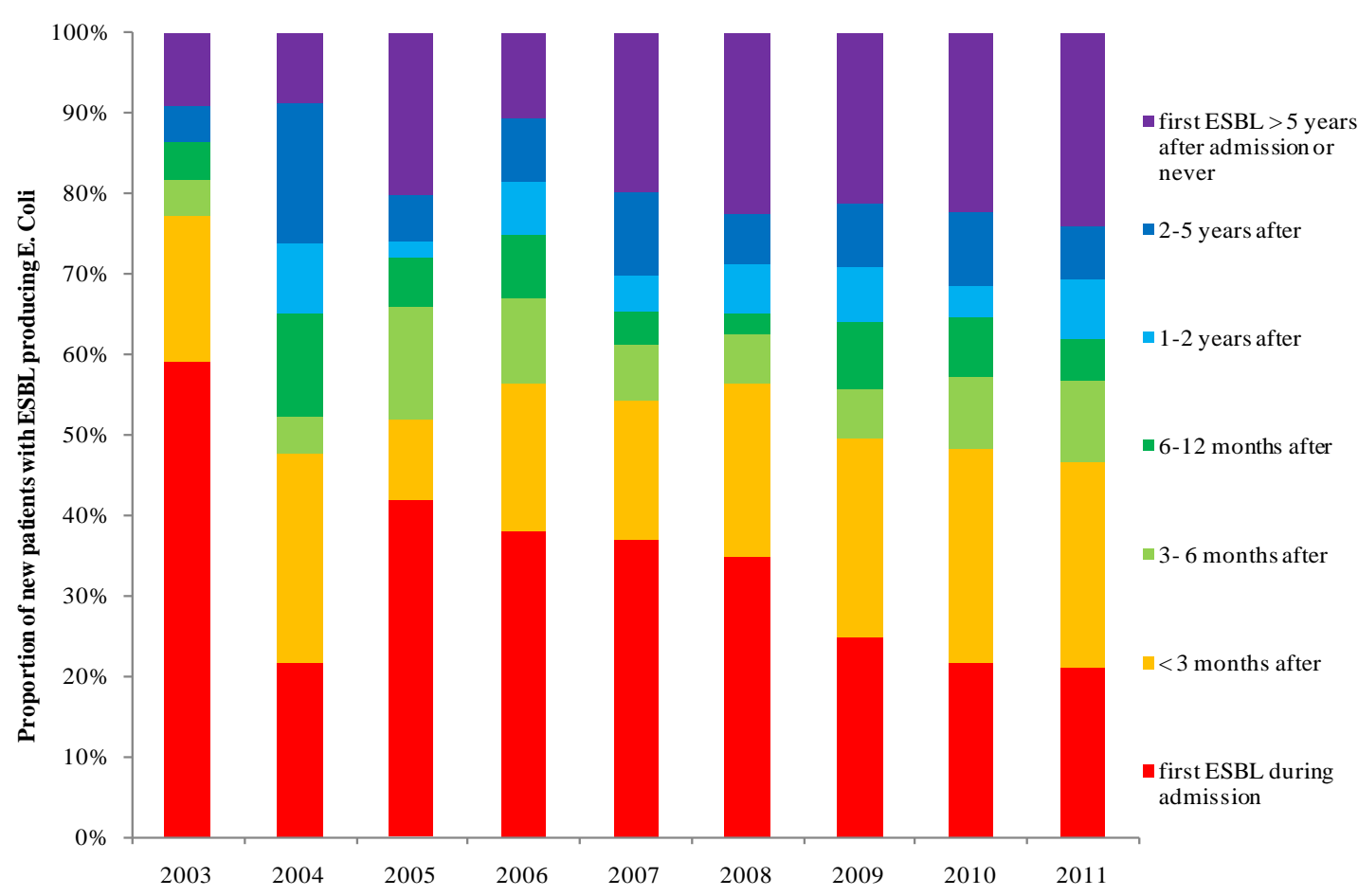

(a)

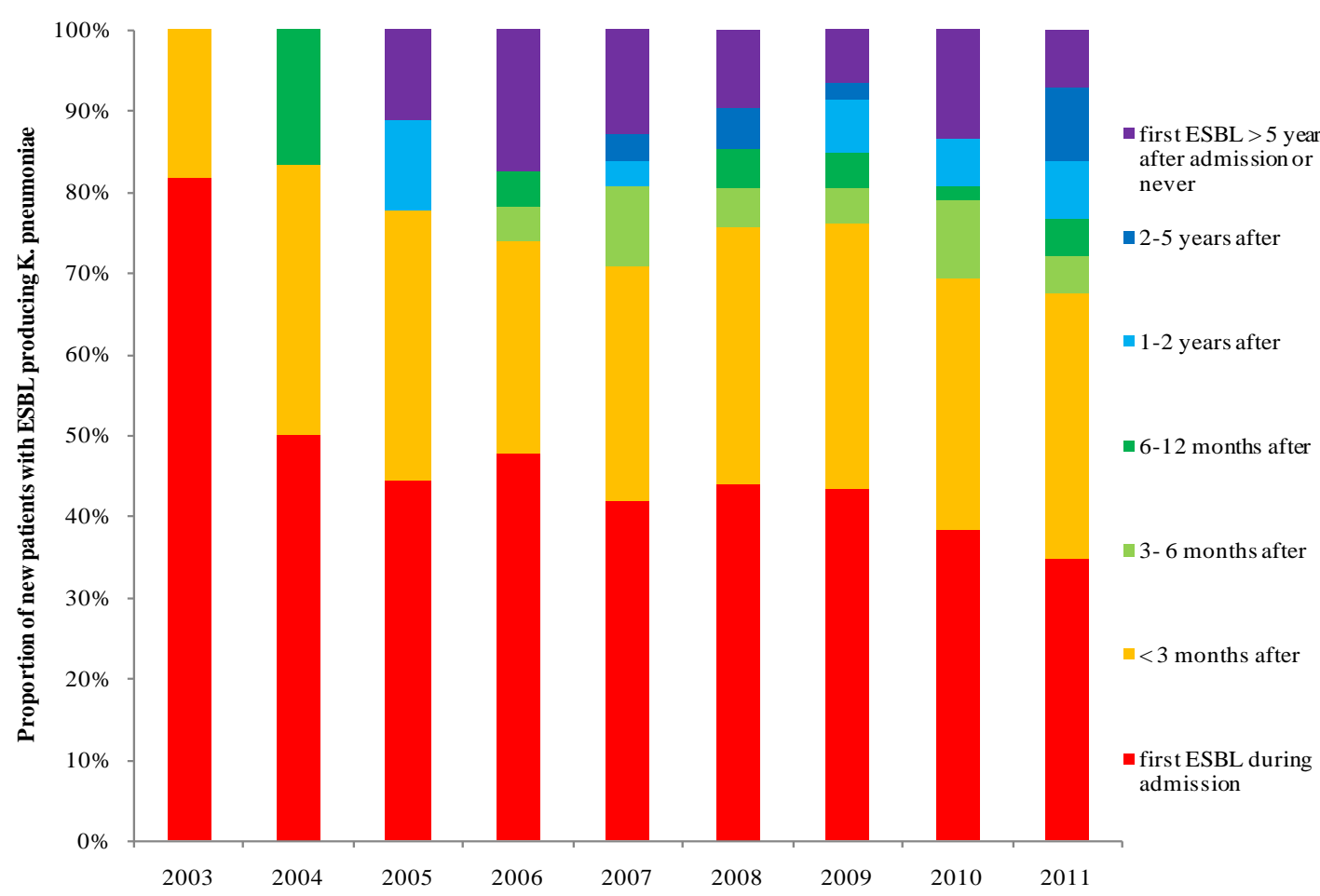

(b)

Figure 2. New patients with an ESBL-producing isolate per year from 2003 to 2011. Columns are divided into the contribution of new ESBL-patients with E. coli (a) and K. pneumoniae (b) in \% according to whether the first finding of an ESBLproducing isolate occurred either under hospitalization or at various intervals after hospital discharge. Markings are given from bottom to top. Red: during hospital admittance; yellow: hospital discharge within 3 months; light green: between 3 and 6 months; dark green: between 6 and 12 months: light blue: between 1 and 2 years; dark blue: between 2 and 5 years; purple: more than 5 years or never. 


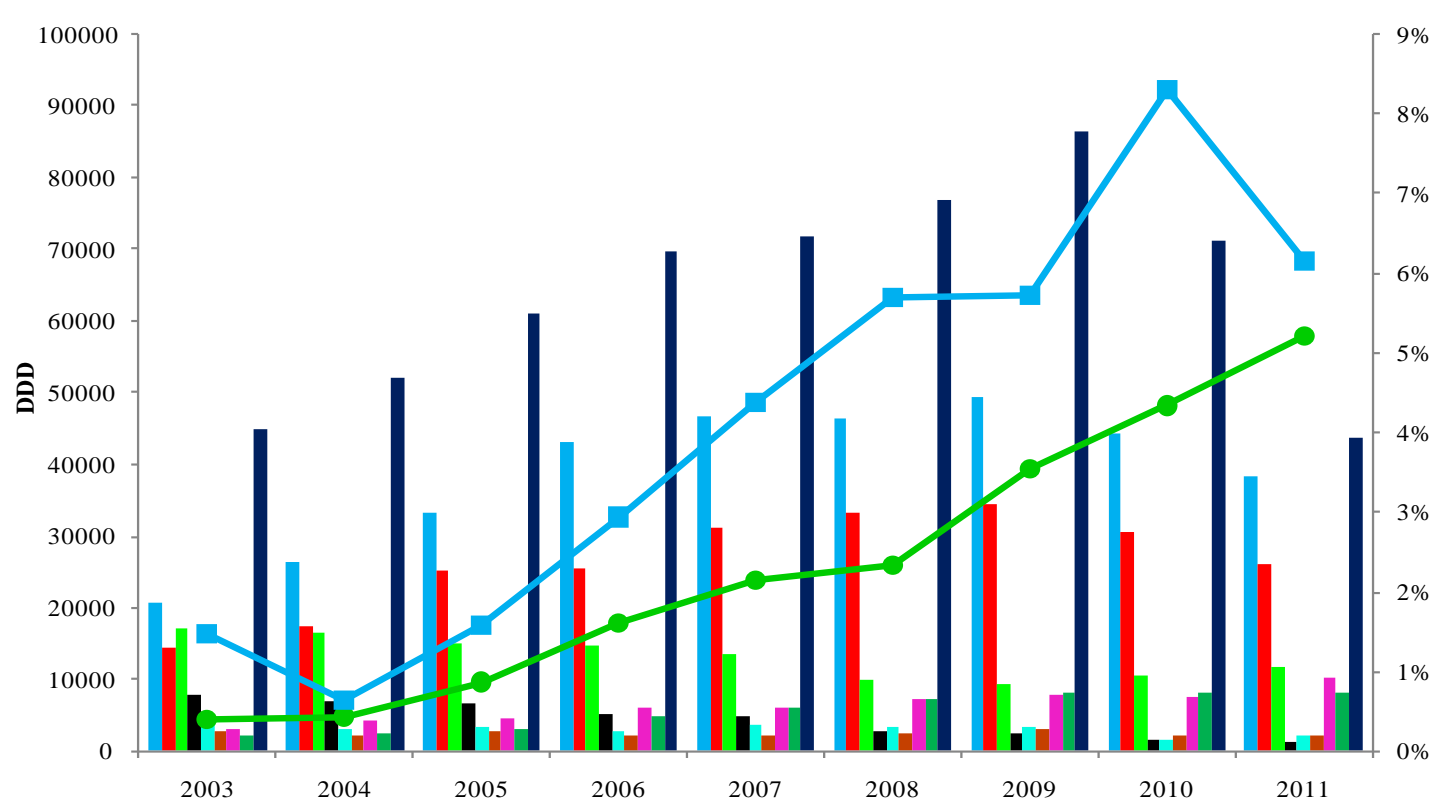

Figure 3. Antimicrobial consumption in DDD (left ordinate) and incidence of ESBL-producing E. coli and K. pneumoniae (right ordinate) from 2003-2011 at the hospitals. Colours of columns are given in relation to the left ordinate from left to right. Light blue: mecillinam; red: ciprofloxacin; light green: gentamicin; black: sulfamethizole; turquoise: trimethoprim; brown: nitrofurantoin; pink: piperacillin with tazobactam; dark green: meropenem; dark blue: cephalosporins. Curves in relation to the right ordinate show patients with an ESBL-producing E. coli in relation to the total number of patients diagnosed with $E$. coli for each year (filled circles); and patients with an ESBL-producing K. pneumoniae in percent of the total number of patients diagnosed with K. pneumoniae for each year (filled squares).

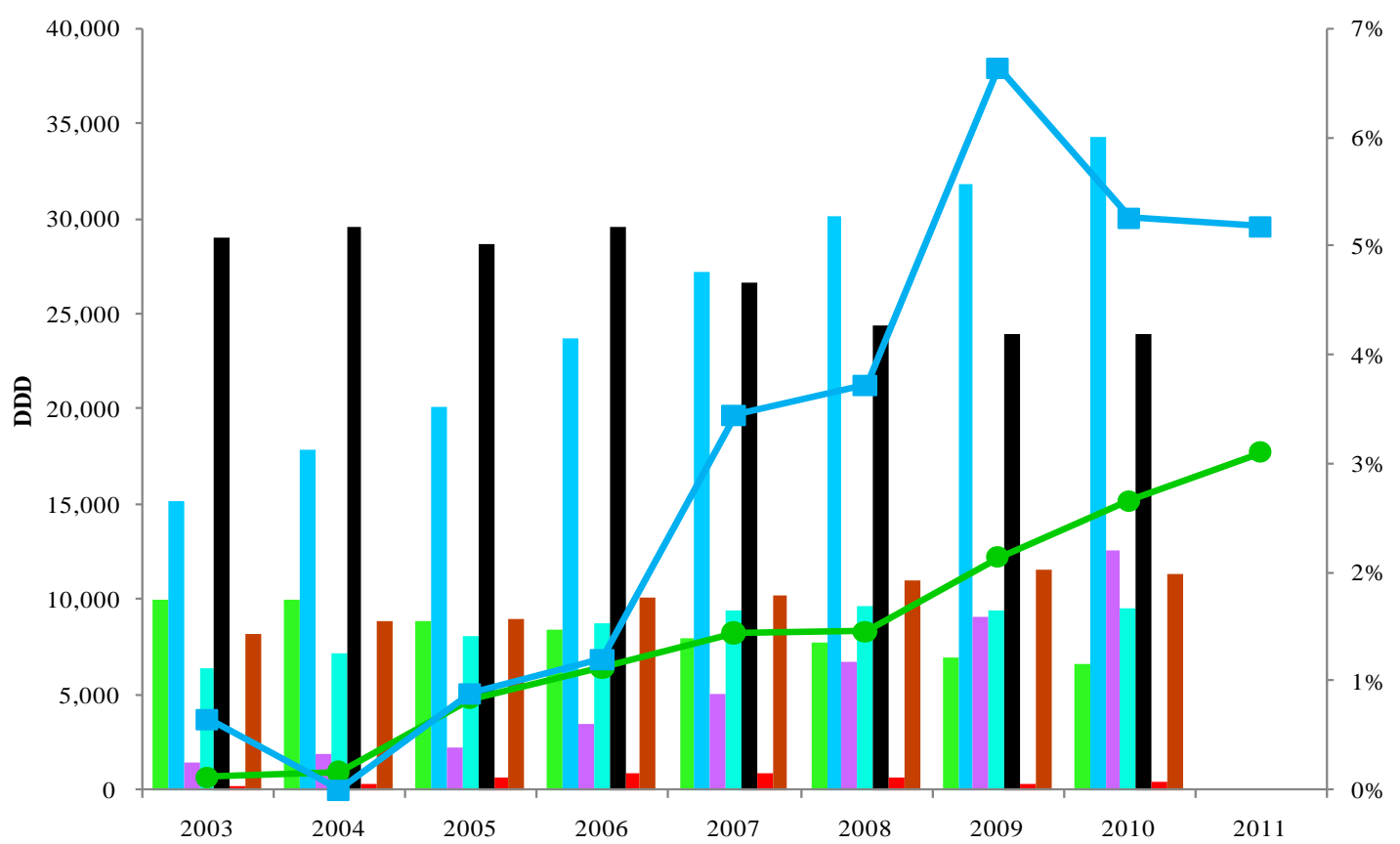

Figure 4. Antimicrobial consumption in DDD (left ordinate) and incidence of ESBL-producing E. coli and K. pneumoniae (right ordinate) from 2003-2010 in primary health care. Colours of columns are given in relation to the left ordinate from left to right. Dark green: pivampicillin; light blue: mecillinam; pink: amoxicillin with clavulanic acid; turquoise: trimethoprim; black: sulfamethizole; red: ciprofloxacin; brown: nitrofurantoin. Curves in relation to the right ordinate show patients with an ESBL-producing $E$. coli in relation to the total number of patients diagnosed with $E$. coli for each year (filled circles); and patients with an ESBL-producing $K$. pneumoniae in relation to the total number of patients diagnosed with K. pneumoniae for each year (filled squares). 


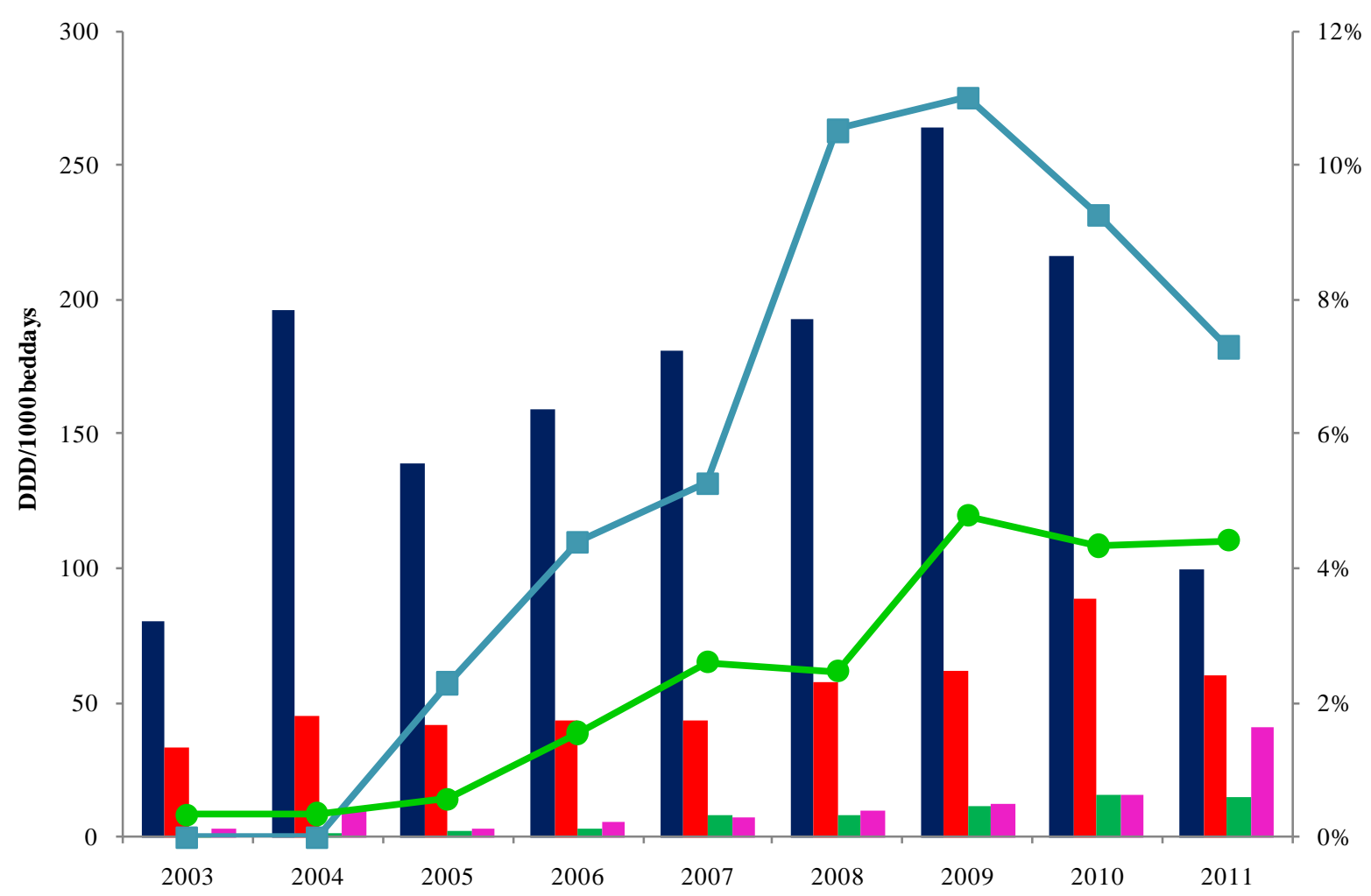

Figure 5. Antimicrobial consumption in DDD and incidence of ESBL-producing E. coli and K. pneumoniae from 2003-2011 at the Department of Internal Medicine at Herlev University Hospital. Colours of columns are given in relation to the left ordinate from left to right. Dark blue: cephalosporins; red: ciprofloxacin; dark green: meropenem; pink: piperacillin with tazobactam. Curves in relation to the right ordinate show patients with an ESBL-producing E. coli in relation to the total number of patients diagnosed with E. coli for each year (filled circles): and patients with an ESBL-producing K. pneumoniae in relation to the total number of patients diagnosed with K. pneumoniae for each year (filled squares).

[17]. Small prospective studies performed in 2001 and 2003 revealed a low prevalence [18]. No national surveillance had taken place in Denmark until a large nationwide prevalence study was done in 2007 and repeated in 2009. These two studies covered 95\% of the Danish population and showed an overall increase in cases of ESBL-producing E. coli bacteraemias from 4.2\% in 2007 to 7\% in 2009 [9], and of ESBL-producing K. pneumoniae bacteraemias from 5\% in 2007 to $14.6 \%$ in 2009. A follow up prevalence study was performed in 2011 and showed a decrease in the number of ESBL-producing E. coli and K. pneumoniae bacteraemia isolates from 2009 to 2011. A small increase (1\%) was, however, seen in the same period in E. coli urine isolates from primary health care and hospitals [10].

The overall increase in incidence of patients with an ESBL-producing E. coli isolate in hospitals and primary health care seen in our study was comparable to the national prevalence studies. From 2010 to 2011 there was a decrease in the incidence of patients with an ESBL-producing $K$. pneumoniae isolate, especially in hospital-acquired cases, the same tendency as seen in the prevalence studies from 2009 to 2011.

As seen in our study there was an increase in antimicrobial consumption of cephalosporins and ciprofloxacin from 2003 to 2009 followed by a gradual decrease in 2009-2011. This change in consumption of broad-spectrum antimicrobials was not due to new guidelines for prescription of antimicrobial agents, but possibly to the concerted effort of clinicians and the Department of Clinical Microbiology. This effort was initiated in 2009 and followed by antibiotic audits with feedback in 2010-2011 in two departments with an initially high incidence of ESBL-producing strains. The incidence of ESBL-producing K. pneumoniae strains decreased remarkably as the consumption of cephalosporins, especially cefuroxime, declined in the Department of Internal Medicine, and to a lesser extent in the Department of Urology. The decrease in consumption of broad-spectrum antimicrobials in the area hospitals coincided with a decrease in the incidence of ESBL-producing $K$. pneumoniae, whereas the incidence of ESBL-producing E. coli continued to increase. The decrease in incidence of ESBL-producing $K$. 
pneumoniae can possibly also be explained by efforts to combat these bacteria in all the hospitals in greater Copenhagen, because transferral of patients between hospitals in the area is very common. Thus, an intervention study performed during 2010 at a neighbouring university hospital with implementation of new guidelines for antibiotic use resulted in a significant decline in ESBL-producing Klebsiella pneumoniae isolates [19]. Several other studies have shown that antibiotic stewardship with a restriction in prescriptions of broad-spectrum antimicrobials, especially cephalosporins and fluoroquinolones, is an important tool in diminishing the incidence of multidrug resistant microorganisms such as ESBL-producing bacteria [20]-[23].

It is difficult to get an exact estimate of the real contribution from primary health care to the incidence of new patients with an ESBL isolate because of the presumed fecal colonization with these bacteria before onset of infection. A very high number of our patients had been hospitalized previously, as described above. In an attempt to further elucidate the role of primary health care, we have presented the proportion of new cases with an ESBL-producing isolate in relation to previous hospitalization by modifying the definition of health care associated bloodstream infection suggested by Friedman et al. [24]. Using these criteria, Ben-Ami et al. [25] found that 65 of 80 patients (81\%) with an ESBL isolate from blood, obtained within two days of admission, to be hospital associated for both K. pneumoniae and E. coli. The study included patients admitted from nursing homes and patients receiving intravenous therapy at home in the group of patients with hospital associated infections. In our study, we were only able to look at previous hospitalization, because we had no data from nursing homes, and intravenous therapy at home is not used in Denmark. The number of patients in hemodialysis with new ESBL isolates was very low and not included in our study. When looking only at the patients with an ESBL-producing K. pneumoniae, 70\% - 80\% of these patients had hospital associated infections, similar to the findings by Ben-Ami et al. [25].

There are not many reports on nosocomial transmission of ESBL-bacteria in hospitals, and as far as we know none on how many patients acquire fecal carriage of ESBL during hospitalization. The few studies of duration of intestinal colonization of ESBLs have found carriage times from three months to more than three years [26]-[30] with a mean carriage time of six months. Kola et al. required three cultures without ESBL-producing bacteria from perirectal screening or stools before declaring a patient free from ESBLs, whereas other studies only used one negative culture. These varying procedures may explain the difference found in the duration of the carrier state. As the duration of the carrier state varies between patients, one would expect an overlap between "true" contributions to the incidence of new cases of community and hospital acquired infections for any time period considered. Despite this expected overlap, a close linear association was found for patients with an new ESBL-producing E. coli isolate more than 24 months after hospital discharge with a slight but steady increase in the contribution from the community to the incidence of ESBL. It is however important to notice that a major contribution to the increase in the incidence of ESBL-producing isolates in our setting presumably comes from the hospitals with regard to both K. pneumoniae and E. coli. For ESBL-producing Klebsiella pneumonia, it was not possible to find any trend in the contribution from primary health care. This may be due to the small number of cases, but it is also possible that the incidence of ESBL-producing Klebsiella pneumoniae follows another epidemiological pattern.

As shown in our study, the resistance patterns in E. coli and K. pneumoniae non-ESBL isolates are rather similar regarding most of the antimicrobial agents. The increase in ciprofloxacin resistant strains is noteworthy, being most pronounced in ESBL strains. The increase in resistance to ciprofloxacin was associated with an increase in consumption of ciprofloxacin at the same time in both hospitals and primary health care. For nonESBL strains the resistance towards aminoglycosides was below 5\% throughout the period. For the ESBL-producing $K$. pneumoniae strains the resistance to gentamicin was associated with the resistance to ciprofloxacin, being $50 \%-65 \%$. This suggests that these two resistance mechanisms are coupled, as described by others [31] [32]. The same phenomenon was not seen in E. coli isolates from 2006 to 2011 (70\% for ciprofloxacin versus $40 \%$ for gentamicin). A high and increasing resistance to trimethoprim from $65 \%$ to $80 \%$ was seen in the ESBL isolates, while the non-ESBL isolates only showed a slight increase. This is in accordance with other studies [33].

It has been argued, that the widely used double diffusion disc method with cephalosporin/cephalosporin and clavulanic acid underreports the incidence of ESBL-producing isolates, because isolates containing genes for both AmpC- and ESBL-enzymes are not identified [34]. The Mast method was therefore introduced in our department in 2009, but only detected a few extra ESBL isolates. This shows that a combination of both resistance mechanisms is not common in Danish isolates at the present time.

During the last 16 years, there has been a national surveillance of consumption of antimicrobial agents in both 
humans and production animals in Denmark. During these years, there has been a steady increase in consumption of broad spectrum antimicrobials, especially cephalosporins and fluoroquinolones in humans [10]. As a consequence of the high and increasing consumption of broad-spectrum antimicrobials with ensuing increasing antibiotic resistance, an antibiotic council was established in 2010. This has resulted in a countrywide plan for reduction of the use of broad-spectrum antimicrobials in humans and animals. Also, the National Board of Health has recently published guidelines for prescription of antimicrobial agents to humans [35].

Some countries, e.g. Sweden and the Netherlands, have established nationwide surveillance programs for ESBLproducing Enterobacteriaceae and guidelines for infection control and prevention [36] [37], but this has not yet been done in Denmark. Great efforts are made on the national and local level to reduce consumption of broadspectrum antibiotics in the hospitals, but much remains to be done in the hospitals and primary health care. Furthermore, more research is essential in this field, especially larger studies concerning long-term faecal carriage of ESBL-producing Enterobacteriaceae.

\section{Conclusion}

The present study showed a coincident increase in patients with an ESBL-producing isolate and consumption of broad-spectrum antimicrobial agents both in hospitals and in primary health care from 2003 to 2009. Interventions to reduce prescription of cephalosporins and ciprofloxacin at the hospitals from 2010 resulted in a remarkable decrease in patients with ESBL-producing $K$. pneumoniae whereas a continuing increase was seen in patients with ESBL-producing E. coli both at hospitals and in primary health care. During the study period, there was only a slight but steady increase in the contribution from the community to the incidence of ESBL-producing E. coli.

\section{Acknowledgements}

We thank: all the technicians at the Department of Clinical Microbiology, Herlev University Hospital, especially H. W. Halberg, for excellent laboratory work. Furthermore, we thank B. Bruun for excellent and critical reading of the manuscript, H. N. Jakobsen from the Capital Region of Denmark for providing data on antimicrobial consumption in primary health care.

\section{Funding}

This study was not supported by any grants.

\section{Transparency Declarations}

None to declare.

\section{References}

[1] Nordmann, P., Naas, T. and Poirel, L. (2011) Global Spread of Carbapenemase-Producing Enterobacteriaceae. Emerging Infectious Diseases, 17, 1791-1798. http://dx.doi.org/10.3201/eid1710.110655

[2] Paterson, D.L. and Bonom,o R.A. (2005) Extended-Spectrum Beta-Lactamases: A Clinical Update. Clinical Microbiology Reviews, 18, 657-686. http://dx.doi.org/10.1128/CMR.18.4.657-686.2005

[3] Pitout, J.D. and Laupland, K.B. (2008) Extended-Spectrum Beta-Lactamase-Producing Enterobacteriaceae: An Emerging Public-Health Concern. The Lancet Infectious Diseases, 8, 159-166. http://dx.doi.org/10.1016/S1473-3099(08)70041-0

[4] Bradford, P.A. (2001) Extended-Spectrum Beta-Lactamases in the 21st Century: Characterization, Epidemiology, and Detection of This Important Resistance Threat. Clinical Microbiology Reviews, 14, 933-951. http://dx.doi.org/10.1128/CMR.14.4.933-951.2001

[5] Bush, K. and Jacoby, G.A. (2010) Updated Functional Classification of Beta-Lactamases. Antimicrobial Agents and Chemotherapy, 54, 969-976. http://dx.doi.org/10.1128/AAC.01009-09

[6] Canton, R. and Coque, T.M. (2006) The CTX-M Beta-Lactamase Pandemic. Current Opinion in Microbiology, 9, 466475. http://dx.doi.org/10.1016/j.mib.2006.08.011

[7] Oteo, J., Perez-Vazquez, M. and Campos, J. (2010) Extended-Spectrum [Beta]-Lactamase Producing Escherichia coli: Changing Epidemiology and Clinical Impact. Current Opinion in Infectious Diseases, 23, 320-326. 
http://dx.doi.org/10.1016/j.mib.2006.08.011

[8] Lytsy, B., Sandegren, L., Tano, E., Torell, E., Andersson, D.I. and Melhus, A. (2008) The First Major Extended-Spectrum Beta-Lactamase Outbreak in Scandinavia Was Caused by Clonal Spread of a Multiresistant Klebsiella pneumoniae Producing CTX-M-15. APMIS: Acta Pathologica, Microbiologica, et immunologica Scandinavica, 116, 302-308. http://dx.doi.org/10.1111/j.1600-0463.2008.00922.x

[9] ESBL in Sweden. http://www.smittskyddsinstitutet.se/statistik/extended-spectrum-beta-lactamase-esbl/

[10] DANMAP (2011) Use of Antimicrobial Agents and Occurrence of Antimicrobial Resistance in Bacteria from Food Animals, Food and Humans in Denmark 2011. http://www.danmap.org/Downloads/ /media/Projekt\%20sites/Danmap/DANMAP\%20reports/Danmap_2011.ashx

[11] Hansen, D.S., Schumacher, H., Hansen, F., Stegger, M., Hertz, F.B., Schonning, K., et al. (2012) Extended-Spectrum Beta-Lactamase (ESBL) in Danish Clinical Isolates of Escherichia coli and Klebsiella pneumoniae: Prevalence, BetaLactamase Distribution, Phylogroups, and Co-Resistance. Scandinavian Journal of Infectious Diseases, 44, 174-181. http://dx.doi.org/10.3109/00365548.2011.632642

[12] NORM/NORM-VET (2011) Consumption of Antimicrobial Agents and Occurrence of Antimicrobial Resistance in Norway. http://www.vetinst.no/eng/Publications/Norm-Norm-Vet-Report/Norm-NormVet-rapporten-2011

[13] Annual Epidemiological Report on Communicable Diseases in Europe 2011. http://www.ecdc.europa.eu/en/publications/Publications/1111_SUR_Annual_Epidemiological_Report_on_Communica ble_Diseases_in_Europe.pdf

[14] (SRGA). TSRGfA. http://www.srga.org/rafmetod/rafmet.htm

[15] EUCAST (2013) TECoAST. http://www.eucast.org/fileadmin/src/media/PDFs/EUCAST_files/Disk_test_documents/EUCAST_Breakpoint_table_v 3.0.pdf

[16] Group M. ESBL and AmpC Detection Disc Sets. http://www.mastgrp.com/Identification\%20strips\%20discs\%20and\%20rings/Glossies/ESBL_6pp.pdf

[17] Hansen, D.S., Sirot, D. and Kolmos, H.J. (1998) Extended Spectrum Beta-Lactamases in Danish Klebsiella Isolates. Ugeskrift for Laeger, 160, 2261-2262.

[18] Kjerulf, A., Hansen, D.S., Sandvang, D., Hansen, F. and Frimodt-Moller, N. (2008) The Prevalence of ESBL-Producing E. coli and Klebsiella Strains in the Copenhagen Area of Denmark. APMIS: Acta Pathologica, Microbiologica, et Immunologica Scandinavica, 116, 118-124. http://dx.doi.org/10.1111/j.1600-0463.2008.00777.x

[19] Andersen, S.E. and Knudsen, J.D. (2013) A Managed Multidisciplinary Programme on Multi-Resistant Klebsiella pneumoniae in a Danish University Hospital. BMJ Quality \& Safety, 22, 907-915. http://dx.doi.org/10.1136/bmjqs-2012-001791

[20] Aldeyab, M.A., Harbarth, S., Vernaz, N., Kearney, M.P., Scott, M.G., Darwish Elhajji, F.W., et al. (2012) The Impact of Antibiotic Use on the Incidence and Resistance Pattern of Extended-Spectrum Beta-Lactamase-Producing Bacteria in Primary and Secondary Healthcare Settings. British Journal of Clinical Pharmacology, 74, 171-179. http://dx.doi.org/10.1111/j.1365-2125.2011.04161.x

[21] Deege, M.P. and Paterson, D.L. (2011) Reducing the Development of Antibiotic Resistance in Critical Care Units. Current Pharmaceutical Biotechnology, 12, 2062-2069. http://dx.doi.org/10.2174/138920111798808301

[22] Hanberger, H., Arman, D., Gill, H., Jindrak, V., Kalenic, S., Kurcz, A., et al. (2009) Surveillance of Microbial Resistance in European Intensive Care Units: A First Report from the Care-ICU Programme for Improved Infection Control. Intensive Care Medicine, 35, 91-100. http://dx.doi.org/10.1007/s00134-008-1237-y

[23] Owens Jr., R.C. and Rice, L. (2006) Hospital-Based Strategies for Combating Resistance. Clinical Infectious Diseases: An Official Publication of the Infectious Diseases Society of America, 42, S173-S181.

[24] Friedman, N.D., Kaye, K.S., Stout, J.E., McGarry, S.A., Trivette, S.L., Briggs, J.P., et al. (2002) Health Care-Associated Bloodstream Infections in Adults: A Reason to Change the Accepted Definition of Community-Acquired Infections. Annals of Internal Medicine, 137, 791-797. http://dx.doi.org/10.7326/0003-4819-137-10-200211190-00007

[25] Ben-Ami, R., Schwaber, M.J., Navon-Venezia, S., Schwartz, D., Giladi, M., Chmelnitsky, I., et al. (2006) Influx of Extended-Spectrum Beta-Lactamase-Producing Enterobacteriaceae into the Hospital. Clinical Infectious Diseases: An Official Publication of the Infectious Diseases Society of America, 42, 925-934. http://dx.doi.org/10.1086/500936

[26] Birgand, G., Armand-Lefevre, L., Lolom, I., Ruppe, E., Andremont, A. and Lucet, J.C. (2013) Duration of Colonization by Extended-Spectrum Beta-Lactamase-Producing Enterobacteriaceae after Hospital Discharge. American Journal of Infection Control, 41, 443-447. http://dx.doi.org/10.1016/j.ajic.2012.05.015

[27] Kola, A., Holst, M., Chaberny, I.F., Ziesing, S., Suerbaum, S. and Gastmeier, P. (2007) Surveillance of Extended- 
Spectrum Beta-Lactamase-Producing Bacteria and Routine Use of Contact Isolation: Experience from a Three-Year Period. The Journal of Hospital Infection, 66, 46-51. http://dx.doi.org/10.1016/j.jhin.2007.01.006

[28] Lohr, I.H., Rettedal, S., Natas, O.B., Naseer, U., Oymar, K. and Sundsfjord, A. (2013) Long-Term Faecal Carriage in Infants and Intra-Household Transmission of CTX-M-15-Producing Klebsiella pneumoniae Following a Nosocomial Outbreak. The Journal of Antimicrobial Chemotherapy, 68, 1043-1048. http://dx.doi.org/10.1093/jac/dks502

[29] Tham, J., Walder, M., Melander, E. and Odenholt, I. (2012) Duration of Colonization with Extended-Spectrum BetaLactamase-Producing Escherichia coli in Patients with Travelers' Diarrhoea. Scandinavian Journal of Infectious Diseases, 44, 573-577. http://dx.doi.org/10.3109/00365548.2011.653582

[30] Zahar, J.R., Lanternier, F., Mechai, F., Filley, F., Taieb, F., Mainot, E.L., et al. (2010) Duration of Colonisation by Enterobacteriaceae Producing Extended-Spectrum Beta-Lactamase and Risk Factors for Persistent Faecal Carriage. The Journal of Hospital Infection, 75, 76-78. http://dx.doi.org/10.1016/j.jhin.2009.11.010

[31] Strahilevitz, J., Jacoby, G.A., Hooper, D.C. and Robicsek, A. (2009) Plasmid-Mediated Quinolone Resistance: A Multifaceted Threat. Clinical Microbiology Reviews, 22, 664-689. http://dx.doi.org/10.1128/CMR.00016-09

[32] Vetting, M.W., Park, C.H., Hegde, S.S., Jacoby, G.A., Hooper, D.C. and Blanchard, J.S. (2008) Mechanistic and Structural Analysis of Aminoglycoside $N$-Acetyltransferase AAC(6')-Ib and Its Bifunctional, Fluoroquinolone-Active AAC(6')Ib-cr Variant. Biochemistry, 47, 9825-9835. http://dx.doi.org/10.1021/bi800664x

[33] van der Donk, C.F., Beisser, P.S., Hoogkamp-Korstanje, J.A., Bruggeman, C.A. and Stobberingh, E.E. (2011) A 12 Year (1998-2009) Antibiotic Resistance Surveillance of Klebsiella pneumoniae Collected from Intensive Care and Urology Patients in 14 Dutch Hospitals. The Journal of Antimicrobial Chemotherapy, 66, 855-858. http://dx.doi.org/10.1093/jac/dkq538

[34] Robberts, F.J., Kohner, P.C. and Patel, R. (2009) Unreliable Extended-Spectrum Beta-Lactamase Detection in the Presence of Plasmid-Mediated AmpC in Escherichia coli Clinical Isolates. Journal of Clinical Microbiology, 47, 358361. http://dx.doi.org/10.1128/JCM.01687-08

[35] Sundhedsstyrelsen (2012) Vejledning om ordination af antibiotika (Only in Danish). http://www.sst.dk/publ/Publ2012/11nov/AntibiotikaOrdvejl.pdf

[36] (2014) Folkhälsomyndigheten ESBL-producerande tarmbakterier Kunskapsunderlag med förslag till handläggning för att begränsa spridningen av Enterobacteriaceae med ESBL. http://www.folkhalsomyndigheten.se/pagefiles/17838/ESBL-producerande\%20tarmbakterier.pdf

[37] (WIP) TDWIP (2005) Measures to Prevent Transmission of Highly Resistant Microorganisms (HRMO). http://www.wip.nl/UK/free_content/Richtlijnen/HRMO.pdf 